(c) 2017 IEEE. Personal use of this material is permitted. Permission from IEEE must be obtained for all other users, including reprinting/ republishing this material for advertising or promotional purposes, creating new collective works for resale or redistribution to servers or lists, or reuse of any copyrighted components of this work in other works. 


\title{
Influence of Design Parameters in the Optimization of Linear Switched Reluctance Motor under Thermal Constraints
}

\author{
Jordi Garcia-Amorós, Member, IEEE, Pere Andrada, Member, IEEE, \\ Balduí Blanque and Marc Marin Genesca
}

\begin{abstract}
The objective of this paper is to present an original study for optimizing the size of the LongitudinalFlux Double-Sided Linear Switched Reluctance Motor (LSRM) under thermal and weight constraints. The performance is evaluated taken into account duty cycle operating conditions and thermal restrictions. The proposed approach couples Finite Element Analysis for magnetic propulsion force computation and Lumped Parameter Thermal Network for thermal transient analysis. The LSRMs design parameters are characterized by the number of phases and by their size denoted by the pole stroke. The operating conditions are the current density, the duty cycle and the admissible temperature rise of the insulation system. The grid search algorithm is used for solving the optimization problem. From the results, with the help of a novel multivariable optimization chart, a set of optimal configurations regarding to miniaturizations and downsizing of LSRMs is provided.
\end{abstract}

Index Terms-Linear Switched Reluctance Motor, Finite Element Method, Thermal Model, Coupled Field Problems.

\section{NOMENCLATURE}

$b_{p} \quad$ Primary pole width $(m)$

$c_{p} \quad$ Primary slot width $(m)$

$T_{p} \quad$ Primary pole pitch $(m)$

$N_{p} \quad$ Number of active poles per side (primary)

$l_{p} \quad$ Primary pole length $(m)$

$b_{s} \quad$ Secondary pole width $(m)$

$c_{s} \quad$ Secondary slot width $(m)$

$D C \quad$ Duty cycle

$\Delta T_{\text {limit }} \quad$ Maximum temperature rise $\left({ }^{\circ} \mathrm{C}\right)$

$T \quad$ Temperature $\left({ }^{\circ} \mathrm{C}\right)$

$t \quad$ Time (s)

$T_{s} \quad$ Secondary pole pitch $(m)$

$N_{s} \quad$ Number of passive poles per side (Secondary)

Manuscript received on December 22, 2016; revised on January 31 2017; accepted March 5, 2017.

J. G. Amorós and M. Marin are with the Electrical Engineering Dept. and Mechanical Engineering Dept. respectively at University Rovira i Virgili, Tarragona (Spain). e-mail: jordi.garcia-amoros@urv.cat; marc.marin@urv.cat

P. Andrada and B. Blanque are with the Electrical Engineering Department at Universitat Politècnica de Catalunya at Barcelona Tech (Spain).e-mail: pere.andrada@upc.edu; blanque@ee.upc.edu $l_{s} \quad$ Secondary pole length $(m)$

$h_{y} \quad$ Primary yoke height $(m)$

$L_{W} \quad$ Lamination stack length $(m)$

$g \quad$ Air gap length $(m)$

PS Pole Stroke $(m)$

$S \quad$ Distance between aligned-unaligned positions $(m)$

$N_{p p} \quad$ Number of poles per phase

$m \quad$ Number of phases

$x \quad$ Translator position $(m)$

$J \quad$ Flat-topped current density peak $\left(A / \mathrm{mm}^{2}\right)$

$M_{\text {Total }}$ Total primary (Stator) mass $(\mathrm{kg})$

$F_{x} \quad$ Electromagnetic propulsion force $(N)$

$F_{X} \quad$ Average electromagnetic force $(N)$

$f_{X C u} \quad$ Average force per unit of copper mass

$f_{X V p} \quad$ Average force per unit of primary steel volume

\section{INTRODUCTION}

INEAR switched reluctance motors (LSRMs) regardless their low force/weight ratio are being focus of increasing interest [1-10] due to their simplicity, robustness and low expected manufacturing costs make them an attractive electric actuator. Despite the fact that LSRMs are simple actuators, they are complex systems whose analysis and design involves physical process of different nature mainly electromagnetic, mechanic and thermal.

In general, coupled field problems can be classified in two types: weak or strong. In weak-coupled problems the effects can be separated and solved by means of a cascade algorithm in which the coupling is performed by updating and transferring thermal field parameters to magnetic field in each iteration $[11,12]$. In the particular case of LSRM, the absence of permanent magnets and the fact that the thermal time constant is much bigger than the electromagnetic one, led to handle the LSRM magneto-thermal problem by means of a weak-coupled field procedure. The temperature dependency of the relative permeability of a non-oriented electrical steel sheet is not significant for temperatures up to $100^{\circ} \mathrm{C}$ (i.e. less than 5\%) and for inductions under $1.4 \mathrm{~T}$ and above $1.7 \mathrm{~T}$ [13]. Therefore, the material property thermal-dependency considered is the electric resistivity from which the copper losses and current density are obtained.

Nowadays, there are a large number of contributions in the technical and scientific literature about optimization methods for solving different types of multivariable problems, ranging from the classical optimization techniques, the linear and non- 
linear programing, to modern methods, such as evolutionary algorithms. A good general description of these methods is given in [14]. In [15] Finite Element Analysis (FEA) and evolutionary computing algorithms for optimizing shapes are combined in applications such as induction-heating. In the field of electric machines, [16] presents a multi-objective optimization procedure for maximizing torque, efficiency and torque density on a 4-phase Switched Reluctance Motor (SRM). In the same sense, [17] pursues optimizing the performance of the Interior Permanent Magnet Synchronous Motors (IPMSM) by minimizing weight and maximizing power output, using a multi-objective optimization procedure which uses a controlled random search algorithm. In [18] three methodologies for the design of IPMSM are compared: Hooke-Jeeves-method, genetic algorithms and grid-search, resulting that grid-search method is the most accurate way to search the optimum provided that the grid is dense enough. This implies a large number of computations so Hooke-Jeeves and genetic algorithms methods should be used for a global search, reducing the search space, and after that a grid-search scheme could be used to find the optimum.

With the purpose of optimizing the design and maximizing the performance of LSRMs this paper proposes a new optimization methodology, based on grid search algorithm that combines two-dimensional Finite Element Analysis (2D FEA) for electromagnetics computations of force and specific forces, and Lumped Parameter Thermal Network (LPTN) for thermal transient response.

The paper is organized as follows. After the introduction that is given in Section II, the optimization methodology is described in section III. The geometrical, FEA and LPTN models of LSRM are presented in Section IV. Section V is dedicated to the description of the optimization algorithm. In Section VI a discussion of the results is shown and finally Section VII outlines conclusions drawn of this research.

\section{Methodology}

The search space is comprised by the geometrical variables $m$ and $P S$ that determines each LSRM configuration and the current density $J$. Those three variables define a triple denoted by $(m, P S, J)$, which can be expressed as a vector of design variables $\chi=\{m, P S, J\}$. The intervals for each one are: $m \in\{2,3,4,5\}$ phases, $P S \in\{3,4,5,6,7,8,9,10\} \quad \mathrm{mm}$ and $J \in\{0 \div 20 ; \Delta J=0.5\} \mathrm{A} / \mathrm{mm}^{2}$, thus $4 \times 8=32$ LSRM machine configurations are analyzed under 41 current density values.

With the aim of downsizing and optimizing the performance of the LSRM, a multi-objective function is defined by the average specific force per unit of copper mass (efficiency), denoted as $f_{X C u}(\mathrm{~N} / \mathrm{kg})$, and the average force per unit of primary steel volume (force density), denoted as $f_{X V p}$ $\left(\mathrm{N} / \mathrm{m}^{3}\right)$. Expressing these functions as set of functions $\mathcal{F}=$ $\left\{f_{X C u}(\chi), f_{X V p}(\chi)\right\}$, the problem can be formulated as: $\max \mathcal{F}(\chi)$ subject to $\mathcal{R}_{i}(\chi) \leq 0, i=1, n_{c}$

where $\mathcal{R}_{i}(\chi)$ are the set of $n_{c}$ constraints, which are in this case the maximum temperature of the electrical insulation system $\left(T_{\text {LIMIT }}\right)$ and the total primary mass $\left(M_{\text {total }}\right)$. This optimization coupled-field problem is solved using the grid search method. This method has a low efficiency in terms of computing time compared with evolutionary algorithms, but the discrete nature of our search space, its size, and the easiness for parallelizing the problem, makes the grid-search methodology an appropriate tool for this study. The proposed methodology combines 2D-FEA for electromagnetic computations of force and specific forces, and Lumped Parameter Thermal Network (LPTN) for thermal transient response. This methodology comprises a 2D-FEA coupled with a LPTN whose results feed an optimization algorithm which finds the optimal configuration subject to given constraints. The 2D-FEA takes as input the vector of design variables $\chi$, returning the averaged propulsion force $F_{X}(\chi)$, the average specific force $f_{X C u}(\chi)$ and the average density force $f_{X V p}(\chi)$. In the same way, the LPTN gives the transient thermal response, which is characterized by the maximum temperature rise $T_{M A X}(\chi)$ and the first order thermal-time constant $K_{T}(\chi)$. The optimization algorithm give rise to a set of optimal configurations for each current density value. These solutions are depicted using a novel multivariable optimization chart, which allow to select visually the best option for each case. Figure 1 shows a block of the proposed methodology.

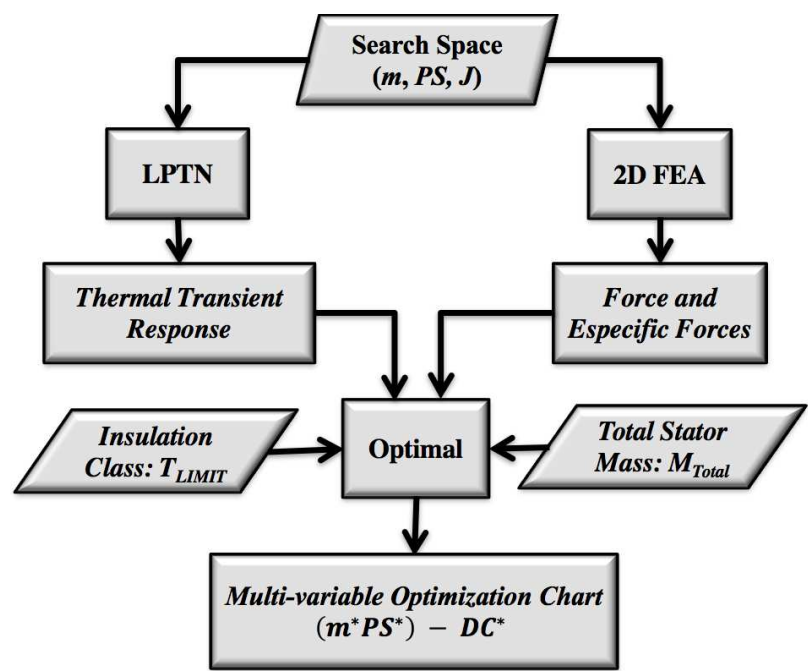

Fig. 1. Block diagram of the proposed methodology

\section{MODELS DESCRIPTION}

\section{A. Geometrical model}

The double-sided flat LSRM considered in this study is made by mirroring a single-sided longitudinal-flux flat LSRM whose result is two primary structures, one on each side, defined as the active part (or primary), we consider this part as the stator of the electrical machine, in which the number of poles per phase $N_{p p}$ is 4 . The secondary (passive part) is made by rectangular poles without connecting iron yokes (see Fig. 2 ), this part is considered as the moving part of the machine, referred as translator or mover.

The input geometrical variables, which characterize the LSRM geometry, are the number of phases $(m)$ and the pole stroke $(P S)$, where it is defined as the distance covered by the secondary from an aligned position to the next aligned 
position when two consecutive phases are excited. Each pair $(m, P S)$ defines a LSRM structure denoted as $\operatorname{LSRM}(m, P S)$. From these two variables, the number of primary and secondary poles $N_{p}$ and $N_{s}$, and pole pitches $T_{p}$ and $T_{s}$ are obtained through equations 2 and 3.

$$
\left.\begin{array}{c}
N_{p}=2 \cdot m \\
N_{s}=2 \cdot(m-1)
\end{array}\right\}
$$

The geometrical parameters (see Fig. 2), associated with the magnetic circuit are: $b_{p}, l_{p}, b_{s} l_{s}$ and $h_{y}$. In order to avoid dimensions, these parameters are normalized to $T_{p}$, resulting a geometrical proportions: $\alpha_{p}=b_{p} / T_{p}, \beta_{p}=l_{p} / T_{p}, \alpha_{s}=b_{s} / T_{p}, \beta_{s}=l_{s} / T_{p}$, $\delta_{y}=h_{y} / T_{p}$, whose values are adopted regarding to optimal force [19]. The air-gap $(g)$ and the stack width $L_{W}$ are held constant. The stator length $L$ (see Fig. 2) can be expressed as:

$$
L(m, P S)=(m-1) \cdot\left[(2 \cdot m-1)+\alpha_{p}\right] \cdot P S
$$

The primary steel volume is:

$$
\begin{aligned}
& V_{p s}(m, P S)=\left[N_{p p} \cdot m \cdot \alpha_{p} \cdot \beta_{p} \cdot L_{W} \cdot(m-1)^{2} \cdot P S^{2}+2 \cdot\right. \\
& \left.\delta_{y} \cdot(m-1) \cdot P S \cdot L_{W} \cdot L(m, P S)\right] \cdot K_{f e}
\end{aligned}
$$

where $K_{f e}$ is the lamination fill factor.

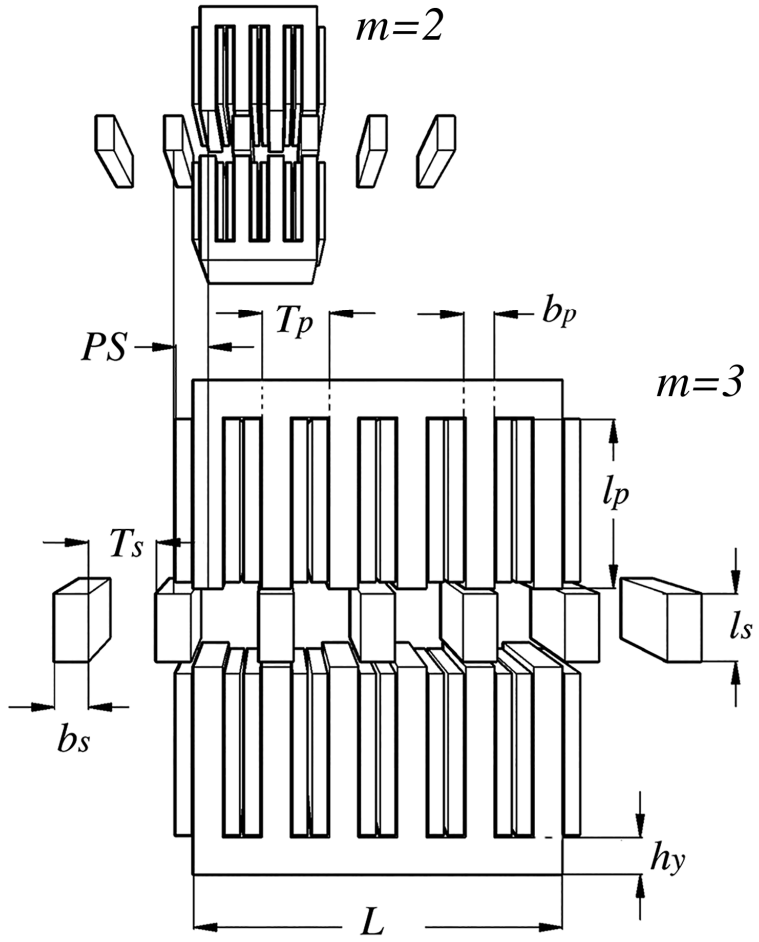

Fig. 2. LSRM main dimensions. $\operatorname{LSRM}(m, \mathrm{PS})$ examples for $m=2$ and $m=3$ at constant PS.

The cooper volume per phase is:

$$
\begin{aligned}
& V_{C u}(m, P S)=m \cdot N_{p p} \cdot K_{S} \cdot \beta_{p} \cdot(m-1)^{2} \cdot P S^{2} . \\
& \left(1-\alpha_{p}\right) \cdot\left[L_{W}+\frac{\pi}{2} \cdot\left(1+\alpha_{p}\right) \cdot(m-1) \cdot P S\right]
\end{aligned}
$$

where $K_{S}$ is the slot fill factor, which it is considered a constant value of 0.4 in this study. The total stator mass can be easily obtained from the addition of copper mass and steel mass (see equations 5 and 6), being: $M_{\text {Total }}=V_{p s}(m, P S)$. $\gamma_{\text {steel }}+V_{C u}(m, P S) \cdot \gamma_{C u}$, where $\gamma_{C u}$ and $\gamma_{\text {steel }}$ are the densities for copper $\gamma_{C u}=8890 \mathrm{~kg} / \mathrm{m}^{3}$ and silicon-iron steel $\gamma_{\text {Steel }}=7750 \mathrm{~kg} / \mathrm{m}^{3}$. Figure 2 shows two 3-dimensional LSRM structures with the same $P S$. The upper picture is for $m=2$ phases denoted as $\operatorname{LSRM}(2, P S)$, and the lower is for $m=3$ phases denoted as $\operatorname{LSRM}(3, P S)$, in which the main dimensions are shown.

\section{B. FEM model}

The 2D-FEM model takes as input parameters the geometrical model $\operatorname{LSRM}(m, P S)$ and the current density $(J)$. From these inputs, the electromagnetic propulsion force $F_{x}$ is obtained by means of the weighted Maxwell stress tensor or eggshell method [20]. This consist on compute the Maxwell stress tensor over a set of concentric surfaces or eggshell shaped region of thickness $\delta$, who encloses the mover and whose contour for each shell is $\Gamma(\delta)$. The computations are averaged over $\delta$, being $n_{y}$ a normal unit vector in $y$-direction (7). These computations are made for several positions evenly distributed between pole misalignment (taken as reference position i.e. $x=0)$ and pole alignment $(x=S=m \cdot P S / 2)$, and averaging over this interval (8).

$$
\begin{aligned}
& F_{x}(m, P S, J, x)=\frac{1}{\delta} \int_{0}^{\delta} \int_{\Gamma(\delta)} \frac{L_{W}}{\mu_{0}} \cdot n_{y} \cdot B_{x} \cdot B_{y} \cdot d \Gamma \cdot d \delta \\
& F_{X}(m, P S, J)=\frac{1}{S} \int_{0}^{S} F_{x}(m, P S, J, x) \cdot d x
\end{aligned}
$$

The average specific forces $f_{X C u}(\mathrm{~N} / \mathrm{kg})$, per unit copper mass, and $f_{X V p}\left(\mathrm{~N} / \mathrm{m}^{3}\right)$, per unit of primary steel volume, are obtained from:

$$
\begin{aligned}
f_{X C u}(m, P S, J) & =\frac{F_{X}(m, P S, J)}{\gamma_{C u} \cdot V_{C u}(m, P S)} \\
f_{X V p}(m, P S, J) & =\frac{F_{X}(m, P S, J)}{V_{p}(m, P S)}
\end{aligned}
$$

Figure 3 shows the FEM model flux lines results for the two structures shown in figure 2, in this case they are not drawn in proportion.

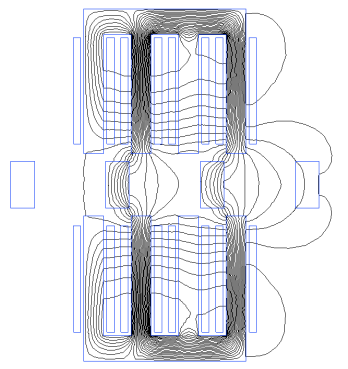

(a)

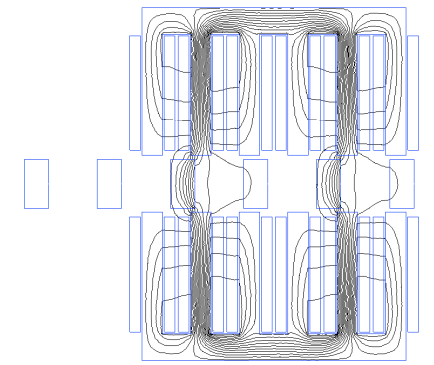

(b)
Fig. 3. 2D-FEM model. a.- LSRM(2,PS). b.- LSRM(3,PS)

\section{LPTN model}

The thermal analysis of electrical machines has been a focus of attention, mainly in the last decade, where there have been many relevant contributions, most of which are collected in reference [21].

In this study case, the thermal approach is carried out by means of a Lumped Parameter Thermal Network (LPTN) under free convection. The LPTN is obtained by reducing the $3 \mathrm{D}$ thermal flow to a $2 \mathrm{D}$ thermal network by lumping parts at the same temperature and being represented by a node. LPTN is similar to an electrical network, in which the nodal voltage [V] corresponds to nodal temperature $[\mathrm{K}]$, the electrical current $[\mathrm{A}]$ corresponds to heat-flow $[\mathrm{W}]$, the electrical 
resistance $[\Omega]$ corresponds to thermal resistance $[\mathrm{K} / \mathrm{W}]$ and finally electrical capacitance $[\mathrm{F}]$ corresponds to thermal capacitance [J/W] [22]. The thermal resistances involved are chiefly due to three heat transfer mechanism: conduction (11), convection (12) and radiation (13) for the different parts of the motor construction. These thermal resistances are computed from the geometrical parameters path-length ( $L$ in $\mathrm{m}$ ) and cross-area $\left(A\right.$ in $\left.\mathrm{m}^{2}\right)$, and from the heat transfers coefficients $k$, $h_{C}$ and $h_{R}$, whose determination is outlined in [23,24] for rotating machines, and in [25] for general cases.

$$
\begin{aligned}
& R_{\text {cond }}=\frac{L}{k \cdot A} \\
& R_{\text {conv }}=\frac{1}{h_{C} \cdot A} \\
& R_{\text {rad }}=\frac{1}{h_{R} \cdot A}
\end{aligned}
$$

The LPTN analysis has been proved a valid tool for thermal design and optimization of electrical actuators, being this technique implemented on commercial software for rotating machines [26]. In the present work the thermal approximation is implemented by a modular thermal network with the ability of expanding for each LSRM structure (see fig.4). The LPTN is defined by four modules in order to model the different $\operatorname{LSRM}(m, P S)$ configurations. Modules 1 and 2 comprises the thermal network for the primary outer and inner poles respectively. Modules 3 and 4 correspond to the primary and secondary iron respectively. The heat source, only due to cooper losses because iron losses are neglected due to the relatively low speed of the LSRM, is modelled as a current source placed in the coil. The thermal resistances are: convection end-winding $\left(R_{e w}\right)$ and lateral surface of outer poles $\left(R_{s w}\right)$, conduction in slot single insulation layer $\left(R_{w}\right)$ and double insulation layer between coils $\left(R_{w w}\right)$, primary's inner surface convection $\left(R_{p}\right)$, inner primary to secondary-iron radiation $\left(R_{p s}\right)$, primary's outer surface combined convectionradiation $\left(R_{c}\right)$, secondary's surface convection $\left(R_{s}\right)$. The nodes are numbered $\{1,2,3,4, \ldots\}$ (see figures $5 \mathrm{a}, 5 \mathrm{~b}$ and $5 \mathrm{c}$ ) and these correspond to nodes of the LPTN shown in figure 4 . The whole LPTN for LSRM set for $m=2$ phases is shown in figure 4. The $C_{u}, C_{p}$ and $C_{s}$ are the thermal capacitances of copper, primary and the secondary iron respectively.

Copper losses for a flat-topped current waveform at an instant $i$ are:

$$
P_{J, i}\left(m, P S, J_{i}, T_{i}\right)=\rho\left(T_{i}\right) \cdot V_{C u}(m, P S) \cdot\left(\frac{J_{i}}{\sqrt{m}}\right)^{2}
$$

where $\rho\left(T_{i}\right)$ is the electrical resistivity at temperature $T_{i}$. The LPTN is implemented and solved by a general purpose software, i.e. MatLab-Simulink, being the LPTN transient response implemented on Simulink and the coupling algorithm on Matlab.

The coupling algorithm major steps are shown in figure 6 . The inputs $m, P S, J$ and $t_{\text {sim }}$ are vectors which contains the search space for each one of the variables, that is, $m=\{2 \div 5\}$ phases, $P S=\{3 \div 10\}-m m, \quad J=\{1 \div 20\}-A / m^{2}$ and $t_{\text {sim }}$ $=\{1 \div$ Period $\}-s$, being the values of $t_{\text {sim }}$ logarithmically spaced.

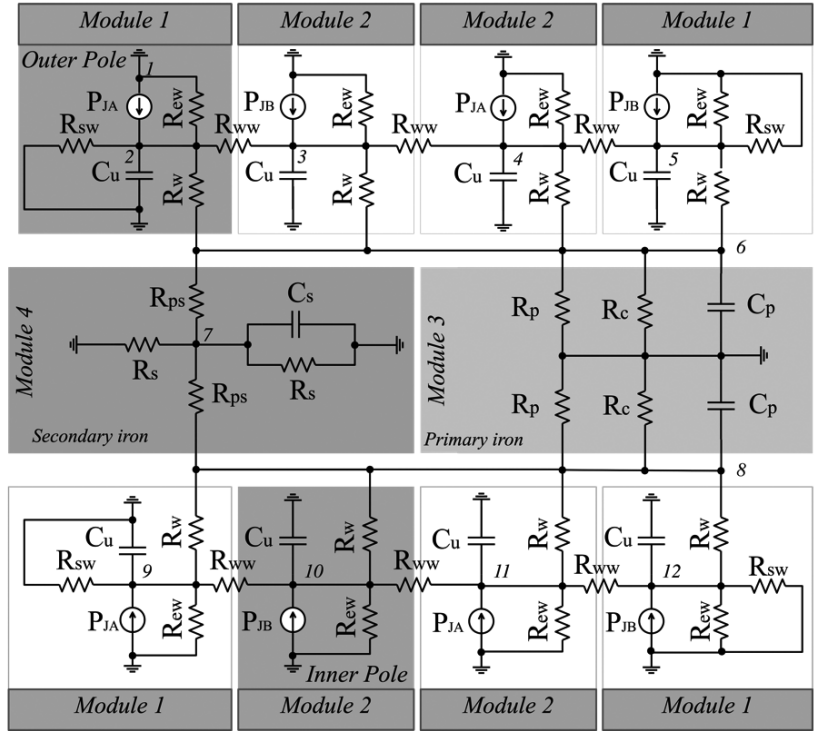

Fig. 4. Thermal equivalent circuit for $\operatorname{LSRM}(2, P S), n(2, P S)=12$ nodes.
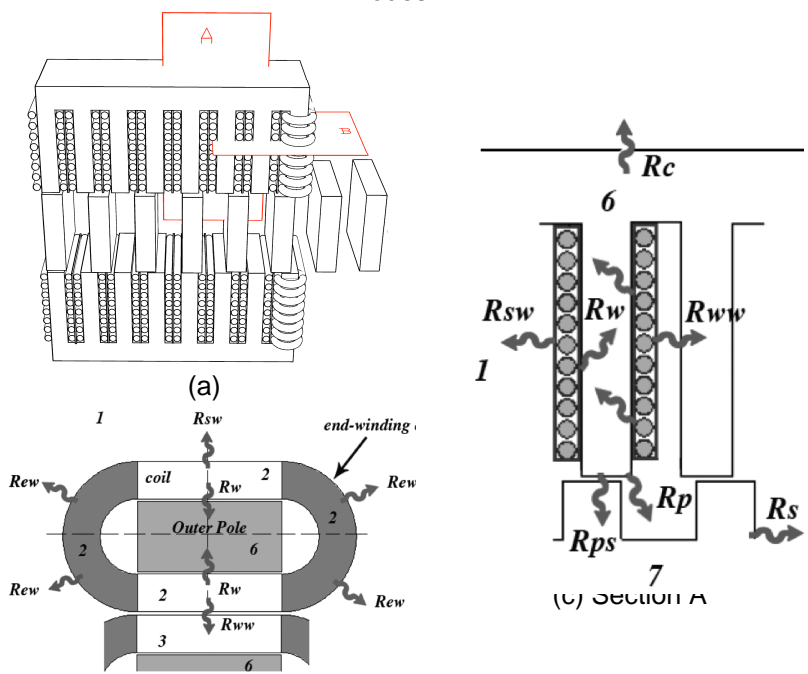

(b) Section B

Fig. 5. Thermal resistances. a) Whole thermal circuit. b) Section B: transversal pole section. c) Section A: longitudinal pole section

The input temperature $T_{0}$ is taken as ambient temperature $\left(40^{\circ} \mathrm{C}\right)$. The subroutine ThermalParam (see fig.6), computes the set of thermal parameters at $T_{i n}$ for each $\operatorname{LSRM}(m, P S, J)$. The subroutine SolveLPTN solves on Simulink the thermal transient analysis and returns the average output temperature after $t_{\text {sim }}[t]$ seconds. In the same sense that in [27], the magneto-thermal weak coupling is implemented in the shadowed box (see Fig. 6), where for each time variation $\Delta t=t \operatorname{sim}[i]-t \operatorname{sim}[i-1]$ it is computed the temperature rise $T_{i}=T_{i-1}+\Delta T$ and from this it is updated the electric resistivity $\rho_{C u}[t+1]$ and the current density $J_{i}[t+1]$, assuming a constant Bus-Voltage operating in single pulse, and finally the copper loses are updated to $P_{J}[t+1]$.

The output of the LPTN is the time-varying temperature for each $\operatorname{LSRM}(m, P S)$ configuration, averaged over the $n$ nodes of the thermal network and fitted to an exponential law (15) with the subroutine FitExp. 


$$
T_{A V G}(m, P S, J, t)=T_{A V G \max }(m, P S, J) \cdot\left(1-e^{\frac{-t}{K_{T}(m, P S, J)}}\right)(15)
$$

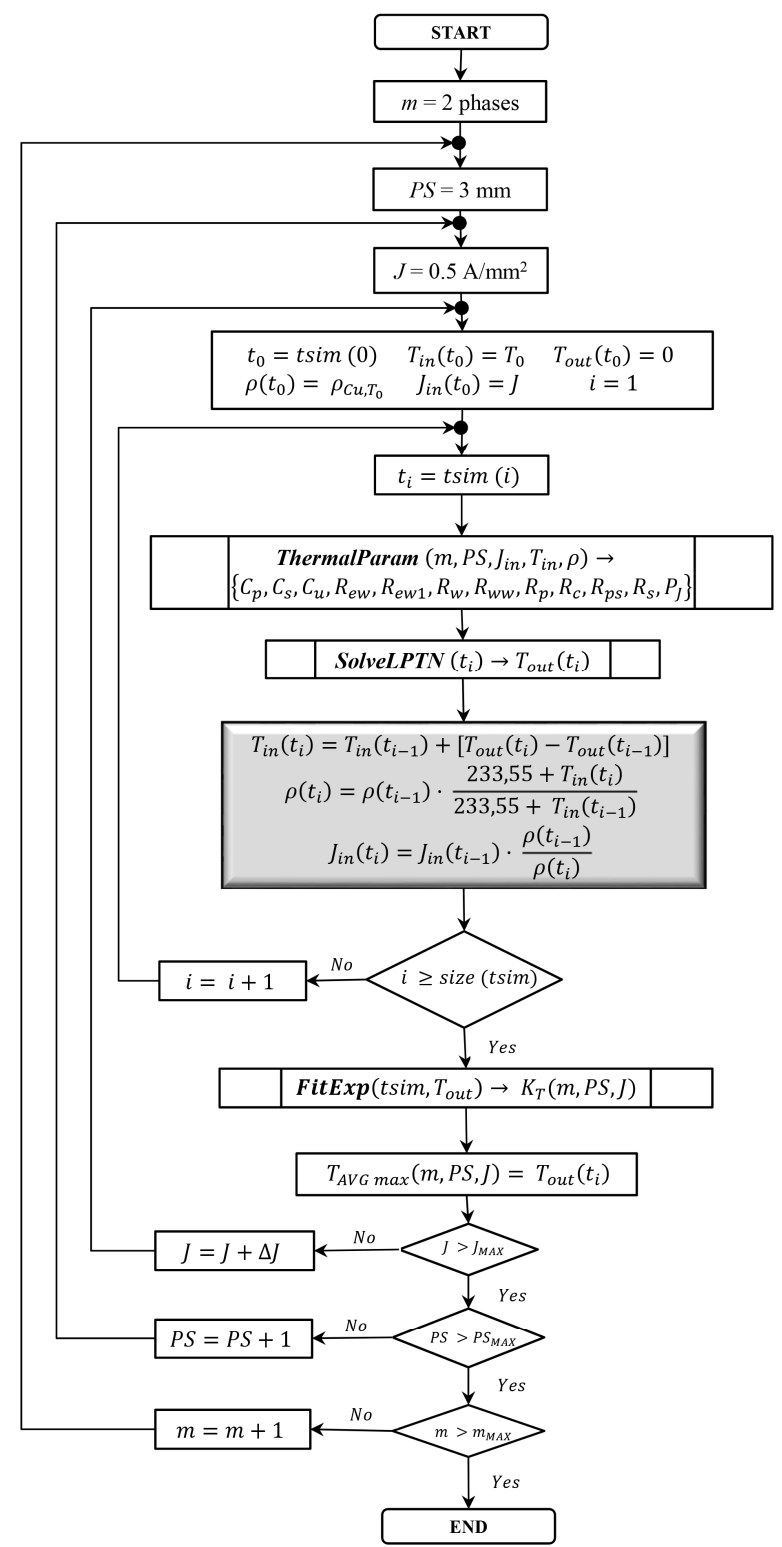

Fig. 6. LPTM coupling algorithm

In general, the LPTN accuracy depends on the network's refinement and the accurate determination of thermal coefficients set $\left\{h_{e w}, h_{w}, h_{w w}, h_{s w}, h_{p}, h_{c}, h_{p s}, h_{s}\right\}$.The main methods used for calculating conduction, radiation, and convection thermal resistances in rotating machines are summarized in $[23,24]$. Due to the flat topology of LSRM these guidelines can be taken as starting values, but is important to adjust the LPTN coefficients by means of a heating test. This calibration consists in adjusting the thermal coefficients set in such way as the LPTN thermal response is as close as possible the heating test results. The heating-test is carried out on a $\operatorname{LSRM}(4,4)$ prototype (see fig.7a). This prototype is built using M270-50 steel grade, 180 wires per coil (24AWG), and assembled under an aluminum frame (see Fig. 7a). The main dimensions are given in table III.
The temperature in the heating test is sensed by 5 temperature sensors (PT100) placed in the end-winding interior gap for each phase and one placed on the top flat plate. The test (see Fig. 7b) reproduces the heating conditions under natural convection due to copper losses for a flat current waveform of $J=10$ and $15 \mathrm{~A} / \mathrm{mm}^{2}$, and for a period of $3600 \mathrm{~s}$. The heating conditions are carried out by feeding all the phases to an effective constant current: $I_{e f}=\frac{\pi \cdot d^{2} \cdot J}{4 \cdot \sqrt{m}}$. Figure 8 shows the LPTN thermal response curve $\left(T_{\text {avg,LPTN FitExp }}\right)(15)$, compared with the thermal test average temperature $\left(T_{\text {avg.test }}\right)$ after calibrating the thermal coefficients. There are some differences (see Fig. 8) at initial values between test measures and LPTN results. This is due to the position of the 4 sensors, placed next to copper winding. At the initial time, the heating up copper's winding can be considered adiabatic [28], and therefore the measures are higher than the average temperature predicted by the LPTN. In [29] a LPTN is calibrated by thermal-test for 4 induction motors of power range from $4 \mathrm{~kW}$ to $55 \mathrm{~kW}$, being the thermal coefficients almost constant. In the same sense, the calibrated thermal parameters are assumed to be constant, and therefore the thermal calibrated values are extended to the rest of $\operatorname{LSRM}(m, P S)$ configurations.

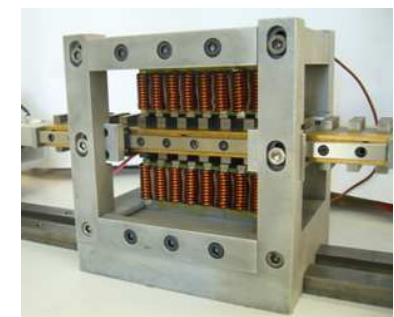

(a)

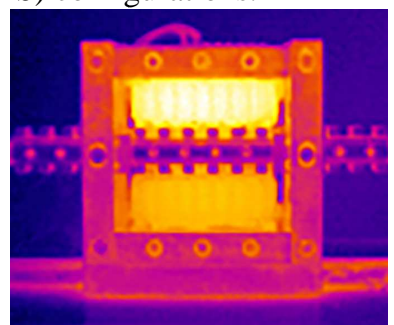

(b)
Fig. 7. (a) LSRM $(4,4)$ prototype. (b) Infrared thermography.

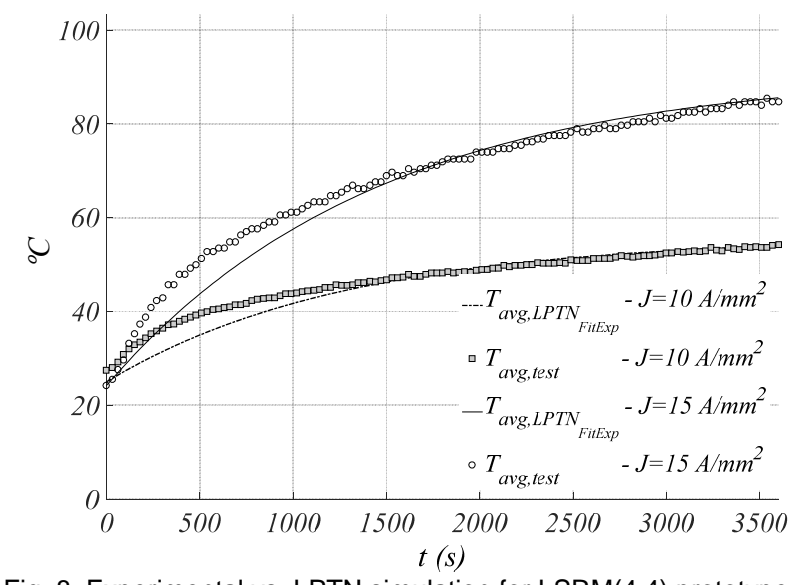

Fig. 8. Experimental vs. LPTN simulation for $\operatorname{LSRM}(4,4)$ prototype.

\section{OptIMIZATION}

The optimization algorithms find the optimal (maximum or minimum) values of a set of mathematical functions that describe a physical problem. In this case, the level of complexity is too high to express the problem by an analytical model or a set of mathematical functions. Therefore, the electromagnetic forces computed by 2D-FEM (8-10) are adjusted to a polynomial, and the LPTN thermal response, is 
adjusted to first order exponential law (15) characterized by the maximum average temperature $T_{A V G \max }$ and the thermal constant $K_{T}$

Our objective is to find the LSRM optimal configurations into the search space variables $\{m, P S, J\}$, subject to a maximum operating temperature restriction defined by the insulation class system and the primary mass. In order to focus the analysis, we center on the particular case of B-Class insulation system, with a limit temperature of $T_{\text {LIMIT }}=120^{\circ} \mathrm{C}$, and the total primary mass under $5 \mathrm{~kg}\left(M_{\text {total }} \leq 5 \mathrm{Kg}\right)$. If the absolute average force $F_{X}$ (see eq.8) is taken as a objective function, the optimization algorithm returns the maximum values of the search space variables, (i.e. $m=5$ phases, $P S=10$ $\mathrm{mm}$ and $J=20 \mathrm{~A} / \mathrm{mm}^{2}$ ) as it is expected, and this is useless for optimizing regarding to miniaturization and downsizing. Hence the objective function that best fits our goal are the average specific forces $f_{X C u}(N / k g)$ and $f_{X V p}\left(N / m^{3}\right)$. Thus, the problem can be reformulated as:

$$
\max \left\{f_{X C u}(m, P S, J), f_{X V p}(m, P S, J)\right\}
$$

Subject to the following restrictions:

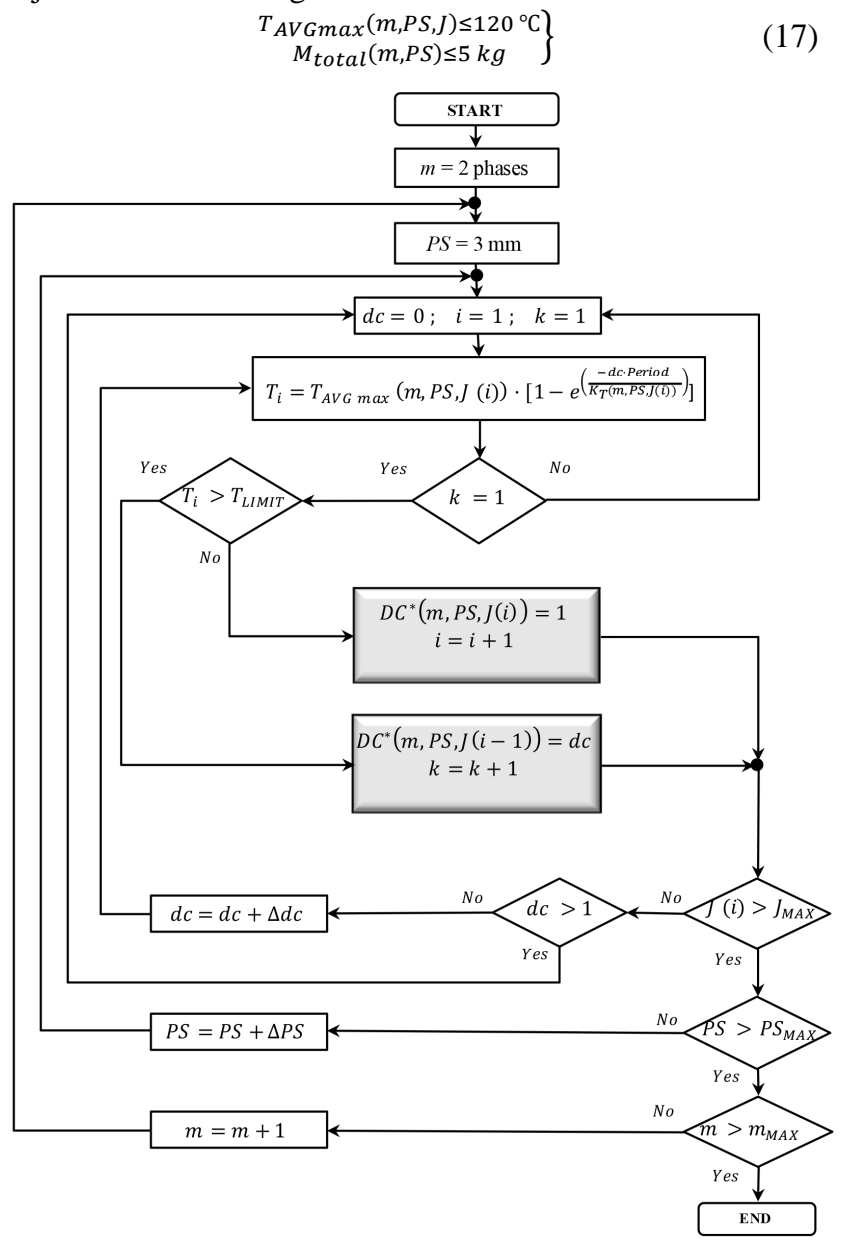

Fig. 9. Optimal algorithm

Figure 9 describes the optimization algorithm major steps. For each $\operatorname{LSRM}(m, P S)$, the time $(d c)$ at which $T_{\text {LIMIT }}$ is reached for a given current density $J$ is computed by using equation 15 ( see fig.9). This time defines the maximum Duty-
Cycle denoted as $D C^{*}$. For each $\operatorname{LSRM}(m, P S, J)$, the maximum average specific forces (9-10) for each value of $J$, referred as $f_{X C u}^{*}$ and $f_{X V p}^{*}$, are searched.

\section{Results AND discussion}

The results of the optimization algorithm (see Fig. 9) are the maximum values of $f_{X C u}^{*}$ and $f_{X V}^{*}$ as function of current density $J$, and the pair $\left(m^{*}, P S^{*}\right)$ at which occurs. The maximum duty cycle $D C^{*}$ for each $\left(m^{*}, P S^{*}, J\right)$ and the total primary mass in addition to. These results are shown in multivariable optimization charts for $f_{X V p}^{*}$ and $f_{X C u}^{*}$, figures 10 and 11 , that allow with a simple glance to have enough information to select the best option for each case.

Despite the fact that the maximum values of $f_{X C u}^{*}$ and $f_{X V p}^{*}$ are at $J=20 \mathrm{~A} / \mathrm{mm}^{2}$ with or without thermal restrictions [30], it is interesting to point some regions out where the optimal configurations $\operatorname{LSRM}(m, P S)$ remain constant for a given current density range. Regarding to miniaturization for applications such as elevator doors or aerospace applications, only configurations with a total mass under $5 \mathrm{~kg}$ are considered (see Table I and II). The configuration $\operatorname{LSRM}(3,10)$ (see Fig. 10 and 11), optimizes both $f_{X C u}$ and $f_{X V p}$, for a current density range from 6.5 to $11 \mathrm{~A} / \mathrm{mm}^{2}$. For higher currents densities there is no optimal in common being $f_{X C u}$ optimal for $m=3$ and $P S=10,9,8$, and $7 \mathrm{~mm}$. The specific force $f_{X C u}$ is also optimal for $m=2$, but this is not considered due to its low absolute force and high ripple force. The specific force $f_{X V p}$ is optimal at $m=3$ and $P S=9,10 \mathrm{~mm}$. Figure 12 shows these optimal LSRM configurations, drawn in proportion. The $\operatorname{LSRM}(3,10)$ configuration has the advantage of being optimal for a wide range of $J$, to reach a maximum average force (3) of $82.6 \mathrm{~N}$ at $J=11 \mathrm{~A} / \mathrm{mm}^{2}$, and to have 3 phases which reduces the converter size and losses.

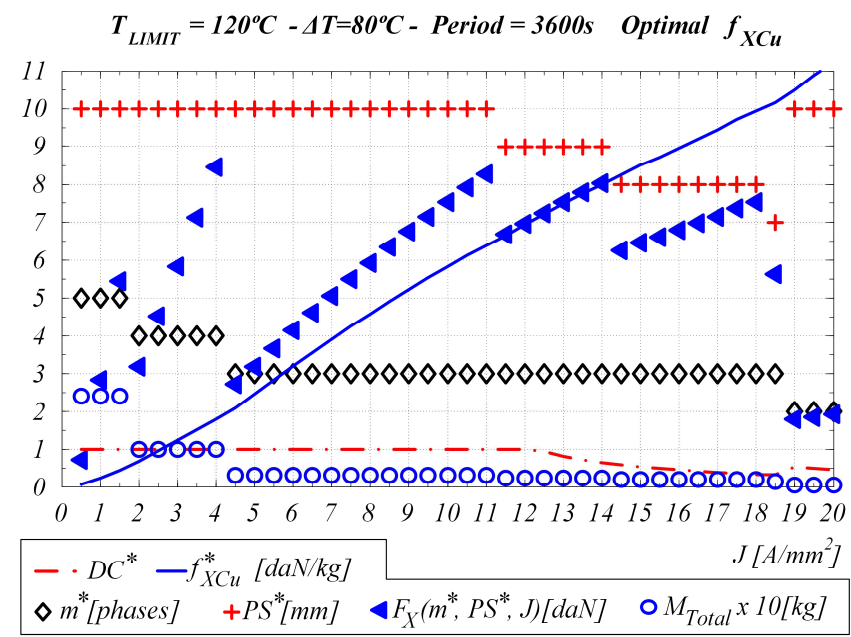

Fig. 10. Multi-variable optimization Chart for $f_{X C u}^{*}\left(m^{*}, P S^{*}, J\right)$ TABLE I

OPTIMAL CONFIGURATIONS FOR $f_{X, C u}$

\begin{tabular}{c|c|c|c|c}
\hline \hline$(m, P S)$ & $J-A / m^{2}$ & $M_{\text {Total }}-k g$ & $F_{X}-N$ & $D C^{*}$ \\
\hline \hline$(3,10)$ & $4.5-11$ & 3.03 & $27.1-82.6$ & 1 \\
\hline$(3,9)$ & $11.5-14$ & 2.4 & $66.7-80.3$ & $1-0.63$ \\
\hline$(3,8)$ & $14.5-18$ & 1.86 & $62.5-75.3$ & $0.59-0.33$ \\
\hline$(3,7)$ & 18.5 & 1.31 & 56.2 & 0.32 \\
\hline \hline
\end{tabular}




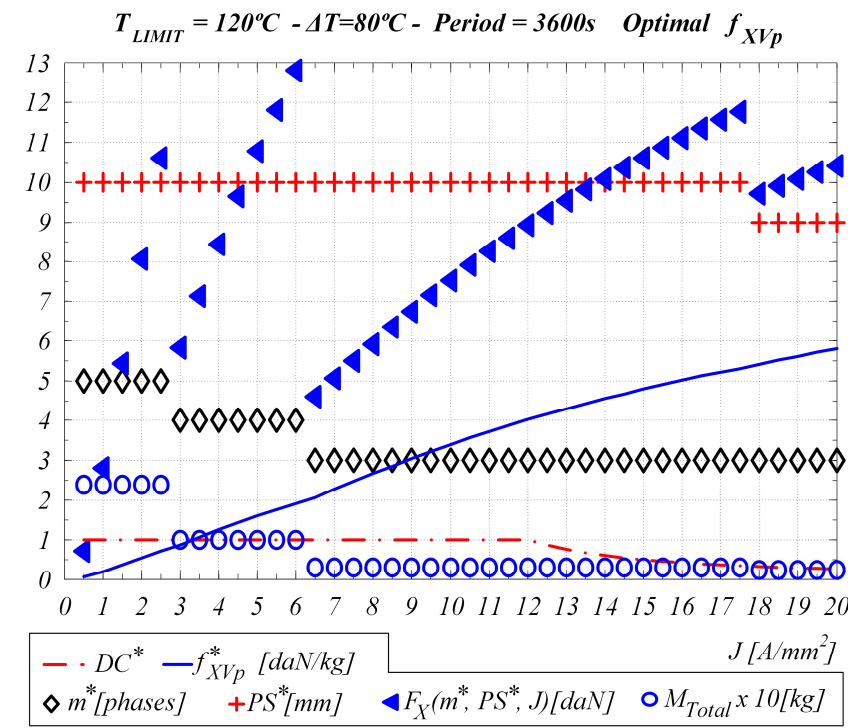

Fig. 11. Multi-variable optimization Chart for $f_{X V p}^{*}\left(m^{*}, P S^{*}, J\right)$

TABLE ॥

OPTIMAL CONFIGURATIONS FOR $f_{X V p}$

\begin{tabular}{c|c|c|c|c}
\hline \hline$(m, P S)$ & $J-A / \mathrm{mm}^{2}$ & \multicolumn{1}{c}{$M_{\text {Total }}-k g$} & $F_{X}-N$ & $D C^{*}$ \\
\hline \hline$(3,10)$ & $6.5-17.5$ & 3.03 & $46-117.7$ & $1-0.33$ \\
\hline$(3,9)$ & $18-20$ & 2.4 & $97.2-104$ & $0.32-0.25$ \\
\hline
\end{tabular}

TABLE III

OPTIMIZED LSRM AND PROTOTYPE MAIN VALUES

\begin{tabular}{|c|c|c|}
\hline & $\begin{array}{c}\operatorname{LSRM}(3,8) \\
\text { Optimized }\end{array}$ & $\begin{array}{c}\text { LSRM(4,4) } \\
\text { Prototype }\end{array}$ \\
\hline$m$ m[phases] & 3 & 4 \\
\hline$P S[\mathrm{~mm}]$ & 8 & 4 \\
\hline$L_{W}[\mathrm{~mm}]$ & 30 & 30 \\
\hline$g[\mathrm{~mm}]$ & 0.5 & 0.5 \\
\hline$T_{p}[\mathrm{~mm}]$ & 16 & 12 \\
\hline$l_{p}[\mathrm{~mm}]$ & 40 & 30 \\
\hline$b_{p}[\mathrm{~mm}]$ & 6.7 & 6 \\
\hline$T_{s}[\mathrm{~mm}]$ & 24 & 16 \\
\hline$l_{s}[\mathrm{~mm}]$ & 8 & 30 \\
\hline$b_{s}[\mathrm{~mm}]$ & 8 & 7 \\
\hline$h_{y}[\mathrm{~mm}]$ & 8.7 & 8 \\
\hline$L[\mathrm{~mm}]$ & 108.4 & 90 \\
\hline$V_{p s}\left[\mathrm{~m}^{3}\right]$ & $1.4187 \cdot 10^{-4}$ & $1.296 \cdot 10^{-4}$ \\
\hline$M_{C u}[\mathrm{~kg}]$ & 0.64 & 0.45 \\
\hline$M_{\text {total }}[\mathrm{kg}]$ & 1.74 & 1.46 \\
\hline \multicolumn{3}{|c|}{ values at $J=15 \mathrm{~A} / \mathrm{mm}^{2}$} \\
\hline$D C^{*}$ & 0.49 & 0.43 \\
\hline$F_{X}[\mathrm{~N}]$ & 64.7 & 25.5 \\
\hline$f_{X C u}[\mathrm{~N} / \mathrm{kg}]$ & 100.5 & 56.4 \\
\hline$f_{X V p}\left[\mathrm{kN} / \mathrm{m}^{3}\right]$ & 456.4 & 196.8 \\
\hline
\end{tabular}

$\operatorname{LSRM}(3,10)$

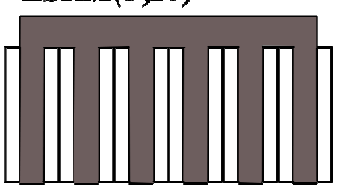

$\operatorname{LSRM}(3,8)$

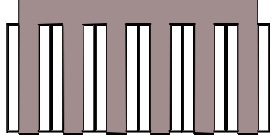

Fig. 12. LSRM optimal configurations for $f_{X C u}$ and $f_{X V p}$

The prototype $\operatorname{LSRM}(4,4)$ used in the thermal calibration test was designed without any optimization procedure. Table III shows the comparison results of the prototype and its optimized version $\operatorname{LSRM}(3,8)$ regarding to the total primary mass $M_{\text {total }}$. As it can be seen this optimization procedure reveals how a $40 \%$ of increasing on copper mass, being almost the same steel mass, produces a significant increase on the average force, about $153 \%$ (see Table III).

Regarding to average propulsion force $\operatorname{LSRM}(3,6)$ gives a force of $F_{X}(3,6,15)=28,7 \mathrm{~N}$ and a total primary mass $M_{\text {total }}=1$ $\mathrm{kg}$. Therefore, a reduction of $31.5 \%$ of the total primary mass produces almost the same thrust. These facts reveal how a low copper and lamination-steel utilization factors can affect the LSRM performance.

\section{CONCLUSIONS}

This paper has presented a coupled FEA-LPTN methodology of analysis for downsizing LSRMs regarding to thermal restrictions $\left(T_{\text {LIMIT }}\right)$ and operating conditions $(J, D C)$ using a grid search optimization algorithm. This methodology has been implemented for assessing a set of $\operatorname{LSRM}(m, P S)$ configurations and has been found the optimal configurations with respect to the average force per unit copper mass and the average force per unit primary steel mass. For the particular case of $T_{\text {LIMIT }}=120^{\circ} \mathrm{C}$ and $M_{\text {total }} \leq 5 \mathrm{Kg}$, the results obtained have revealed a set of optimal configurations, being the $\operatorname{LSRM}(3,10)$ optimal for both $f_{X C u}$ and $f_{X V p}$, in a current density range from 6.5 to $11 \mathrm{~A} / \mathrm{mm}^{2}$.

\section{REFERENCES}

[1] Wenlong Li; Chau, K.T.; Chunhua Liu; Chun Qiu, "Design and Analysis of a Flux-Controllable Linear Variable Reluctance Machine", IEEE Transactions on Applied Superconductivity, vol. 24, no. 3, pp. 1-4, June 2014

[2] Chen, H.; Sun, C.; Wang, Q., "Analysis of Flux-Linkage Characteristics of Switched Reluctance Linear Generator", IEEE Transactions on Applied Superconductivity, vol. 24, no. 3, pp. 1-5, June 2014.

[3] Song S., Zhang M., Ge L., Wang L., "Multiobjective Optimal Design of Switched Reluctance Linear Launcher", IEEE Transactions on Plasma Science, vol. 43, no. 5, pp. 1339-1345, May 2015.

[4] Garcia-Amorós J., Bargalló-Perpiñà R., Andrada P., Blanqué B., "Thermal performance analysis of the double-sided linear switched reluctance motor", International Conference on Electrical Machines (ICEM), pp. 1448-1454, 6-8 September 2014, Berlin (Germany),

[5] Wang D., Shao C., Wang X., "Performance Analysis and Design Optimization of an Annular Winding Bilateral Linear Switch Reluctance Machine for Low Cost Linear Applications" in IEEE 
Transactions on Applied Superconductivity, vol. 26, no. 7, pp. 1-5, Oct. 2016

[6] Lin J., Cheng K. W. E., Zhang Z., Cheung N. C., Xue X., “Adaptive sliding mode technique-based electromagnetic suspension system with linear switched reluctance actuator", IET Electric Power Applications, vol. 9, no. 1, pp. 50-59, 12015.

[7] Zhao S. W., Luo L. N., Yang L. Q., Cheung N. C. "High precision position control of Linear Switched Reluctance Motor for short distance" 5th Int. Conf. on Power Electronics Systems and Applications (PESA), 2013, Hong Kong, 2013, pp. 1-5.

[8] Pan J. F., Zou Y., Cao G., Cheung N. C., Zhang B., "High-Precision Dual-Loop Position Control of an Asymmetric Bilateral Linear Hybrid Switched Reluctance Motor" IEEE Transactions on Magnetics, vol. 51, no. 11, pp. 1-5, Nov. 2015.

[9] Lobo N. S., Lim H. S., Krishnan R., "Comparison of Linear Switched Reluctance Machines for Vertical Propulsion Application: Analysis, Design, and Experimental Correlation", IEEE Transactions on Industry Applications, vol. 44, no. 4, pp. 1134-1142, July-Aug. 2008

[10] Dursun M., Özbay H., "Design and analysis of a double sided linear switched reluctance motor driver for elevator door", Przeglad Elektrotechniczny, R. 87 NR 5/2011

[11] Hameyer, K.; Driesen, J.; De Gersem, H.; Belmans, R., "The Classification of Coupled Field Problems", IEEE Transactions on Magnetics, vol. 35, no. 3, pp. 1618-1621, May 1999.

[12] Behjat V. A., "Coupled Thermal-Electromagnetic FEM Model to Characterize the Thermal Behaviour of Power Transformers Damaged By Short Circuit Faults", International Journal of Electrical Energy, Vol. 1, No. 4, December 2013

[13] Takahashi N., Morishita M., Miyagi D., Nakano M., "Examination of Magnetic Properties of Magnetic Materials at High Temperature using a Ring Specimen”, IEEE Trans. Magn., 46 (2010), No. 2, 548-551.

[14] Rao S. S. Engineering Optimization: Theory and Practice. 4th Edition. John Wiley \& Sons 2009.

[15] Di Barba P., Dolezel I., Mognaschi M. E., Savini A., Karban P., "NonLinear Multi-Physics Analysis and Multi-Objective Optimization in Electroheating Applications", IEEE Transactions on Magnetics, vol. 50, no. 2, pp. 673-676, Feb. 2014.

[16] Xue X. D., Cheng K. W.E., Ng T. W. Cheung N. C. "Multi-Objective Optimization Design of In-Wheel Switched Reluctance Motors in Electric Vehicles", IEEE Transactions on Industrial Electronics, vol. 57, no. 9, pp. 2980-2987, September 2010.

[17] Parasiliti F., Villani M., Lucidi S., Rinaldi F., "Finite-Element-Based Multiobjective Design Optimization Procedure of Interior Permanent Magnet Synchronous Motors for Wide Constant-Power Region Operation", IEEE Transactions on Industrial Electronics, vol. 59, no. 6, pp. 2503-2514, June 2012.

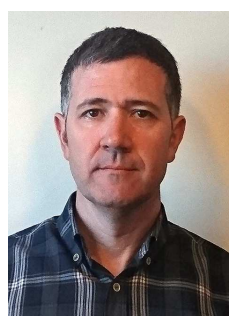

Jordi Garcia Amoros (M'09) was born in Barcelona, Spain in 1970. He received the Ph.D. degree in Electrical Engineering from Universitat Politècnica de Catalunya, Barcelona, Spain in 2010. Since 1992, he has been with the Department of Electrical Engineering at University Rovira i Virgili, in Tarragona Spain, first as Assistant in electrical machines, and since 2014 as Associate Professor. His research interest are in the field of electrical machines. of electromagnetic design and thermal analysis

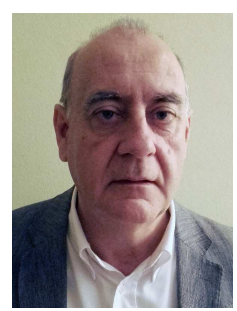

Pere Andrada (M'91) was born in Barcelona (Spain) in 1957. He received the $\mathrm{PhD}$. degree in Industrial Engineering from the Universitat Politècnica de Catalunya, Barcelona, Spain, in 1990. In 1980 he joined the Department of Electrical Engineering, Universitat Politècnica de Catalunya, where he is currently Associate Professor in the Escola Politècnica Superior d'Enginyeria de Vilanova i la Geltrú. He is member of the Electronically Commutated Drive Group. His teaching activities and research interests include design, modeling and control of electrical machines and drives.
[18] Iles-Klumpner D., Boldea I., "Comparative Optimization Design of an Interior Permanent Magnet Synchronous Motor for an Automotive Active Steering System", IEEE 35th Annual Power Electronics Specialists Conference, 2004, pp. 369-375, vol.1.

[19] Amoros, J. G.; Andrada, P., "Sensitivity Analysis of Geometrical Parameters on a Double-Sided Linear Switched Reluctance Motor", IEEE Transactions on Industrial Electronics, vol. 57, no. 1, pp. 311319, January 2010.

[20] Henrotte, F. Deliège, G. Hameyer, K., "The eggshell method for the computation of electromagnetic forces on rigid bodies in 2D and 3D". CEFC 2002, Perugia, Italy, 16-18 April 2002.

[21] Boglietti A., Cavagnino A., Staton D., Shanel M., Mueller M., Mejuto C., "Evolution and Modern Approaches for Thermal Analysis of Electrical Machines",IEEE Transactions on Industrial Electronics, vol. 56, no. 3, pp. 871-882, March 2009.

[22] Amoros J.G., Andrada P., Blanque B. "An Analytical Approach to the Thermal Design of a Double-Sided Linear Switched Reluctance Motor". IX Int. Conf. on Electrical Machines (ICEM), pp. 1-4, 6-8 September 2010. Rome (Italy)

[23] Staton D. A., Cavagnino A., "Convection Heat Transfer and Flow Calculations Suitable for Electric Machines Thermal Models", IEEE Transactions on Industrial Electronics, vol. 55, no. 10, October 2008.

[24] Boglietti A.; Cavagnino A.; Staton, D., "Determination of Critical Parameters in Electrical Machine Thermal Models", IEEE Transactions on Industry Applications, vol. 44, no. 4, pp. 1150-1159, July-Aug. 2008

[25] Incropera F. P., DeWitt D. P., Bergman T. L., Lavine A. S., Fundamentals of Heat and Mass Transfer. 6th Edition. John Wiley \& Sons. 2007

[26] Motor-CAD Thermal solver. [Online]. Available: www.motordesign.com.

[27] Mezani S., Takorabet N., Laporte B., "A combined electromagnetic and thermal analysis of induction motors", IEEE Transactions on Magnetics, vol. 41, no. 5, pp. 1572-1575, May 2005.

[28] Boglietti A., Carpaneto E., Cossale M., Vaschetto S., "Stator-Winding Thermal Models for Short-Time Thermal Transients: Definition and Validation", IEEE Transactions on Industrial Electronics, vol. 63, no. 5, pp. 2713-2721, May 2016.

[29] Boglietti A., Cavagnino A., Staton D. A., "TEFC induction motors thermal models: a parameter sensitivity analysis", Industry Applications Conference, 2004. 39th IAS Annual Meeting. Conference Record of the 2004 IEEE, pp. 2469-2476 vol.4.

[30] Garcia-Amoros J, Andrada P., Blanqué B., "Assessment of Linear Switched Reluctance Motor's Design Parameters for Optimal Performance", Electric Power Components and Systems. Vol. 43, pp. 810-819, Iss. 7, 2015.

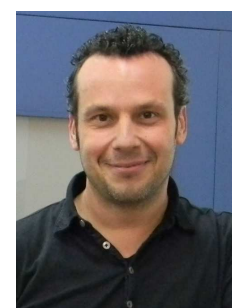

Balduí Blanqué was born in Reus (Tarragona, Spain) in 1970. He received the Ph.D. degree from the Universitat Politècnica de Catalunya, in Barcelona, Spain, 2007. Since 1996, he has been with the Department of Electrical Engineering, UPC, where he is currently an Associate Professor at Escola Politècnica Superior d'Enginyeria de Vilanova i la Geltrú. $\mathrm{He}$ is member of the Electronically Commutated Drives Group. His teaching activities cover digital design and electronics applications and his research interests include modeling, simulation and control of electrical machines and drives.

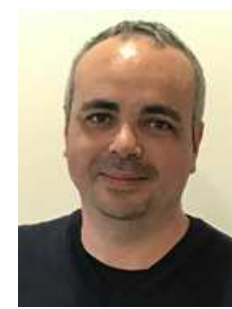

Marc Marín Genescà, was born in Matadepera (Barcelona), Spain in 1976. He received the Ph.D. degree in Electrical Engineering from Polytechnic University of Catalunya, Terrassa (Barcelona), Spain in 2011. Since 2015, he has been with the Department of Mechanical Engineering at University Rovira i Virgili, Tarragona Spain, as Visiting Professor. His research interest are in the field of the characterization of dielectric materials and electromagnetic design and thermal analysis of electrical machines. 